\title{
OBSERVATIONS ON PHOTOCHEMICAL REACTIONS IN LIVING EYES*
}

\author{
BY \\ R. A. WEALE \\ Visual Research Division, Medical Research Council Ophthalmological Research Unit, \\ Institute of Ophthalmology, London
}

\section{Introduction}

LIGHT entering an eye loses some of its intensity as it traverses the ocular media and the retina. Part of the light reaching the fundus is reflected, retraverses the retina and the ocular media, and emerges from the pupil. The intensity of the emerging beam can be measured: its ratio to the intensity of the light originally entering the eye $\left(R_{\lambda}\right)$ depends on reflection losses at the ocular interfaces and at the fundus, on losses due to scattering, and on absorption losses. In particular, it is possible to record changes in $\boldsymbol{R}_{\lambda}$ as due to changes in any of these factors. The eye having been in the dark for about an hour, $R_{\lambda}$ can be measured with various monochromatic lights of low intensity. When the eye is subsequently exposed to light from a strong source, photo-sensitive, light-absorbing material, which accumulated in the retina during dark-adaptation, is bleached. If the same monochromatic radiations are used to re-measure the fraction of light $R_{\lambda}$ emerging from the eye, $R_{\lambda}$ is generally found to have increased: more light emerges from the eye because the act of bleaching has removed light-absorbing material. The change in $R_{\lambda}$, expressed as $\Delta \log R_{\lambda}$ can be plotted against the wavelength $\lambda$, and a difference spectrum obtained. Its shape and spectral location is characteristic of the photo-sensitive material removed by the bleaching radiation.

The emphasis has recently been, not unnaturally, on the characterization of visual pigments in living eyes rather than on a study of their behaviour as a result of the exposure to light. There is little doubt, however, that, from the point of view of understanding vision, a knowledge of the behaviour of pigments immediately after exposure to light is important. Three brief notices are of interest in this connexion. Wulff, Abrahamson, Adams, and Linschitz (1956) examined the densitometric properties of cattle visual purple solutions by flash photolysis combined with spectro-photography; Hagins (1956) followed the time course of the retinal pigment density in enucleated rabbit eyes; Weale (1956a) used a spectro-photographic method in the study of pigments in living cat and rabbit eyes, and this is described in greater detail in the second part of this paper. The first part, however, deals

* Received for publication February 22, 1957. 
with some earlier observations made on the behaviour of cat (Weale,1953a, b) and frog retinae after the eye had been exposed to white light, a photometric method (Weale, 1951, 1953c) of measuring $R_{\lambda}$ being used. These experiments first revealed the need for examining eyes with the greatest possible speed.

\section{Method A}

The apparatus consisted of a Hilger Barfit monochromator and a photometer (Fig. 1). The monochromator was illuminated with a tungsten ribbon filament lamp, Ilford bright spectrum filters Nos. 622-6, 607-8 being used to reduce the effect of scattered light. Light emerging from the exit-slit $\mathrm{S}$ of the monochromator was rendered parallel by the photometer lens $L_{1}$. The photometer prism $(P)$, half of a diagonal plane of which was silvered, divided the beam into two equal parts. One of these, the matching beam, was focused by the lens $L_{2}$ on to a magnesium oxide surface $M$. The other, the testing-beam, was reflected by the silvered part

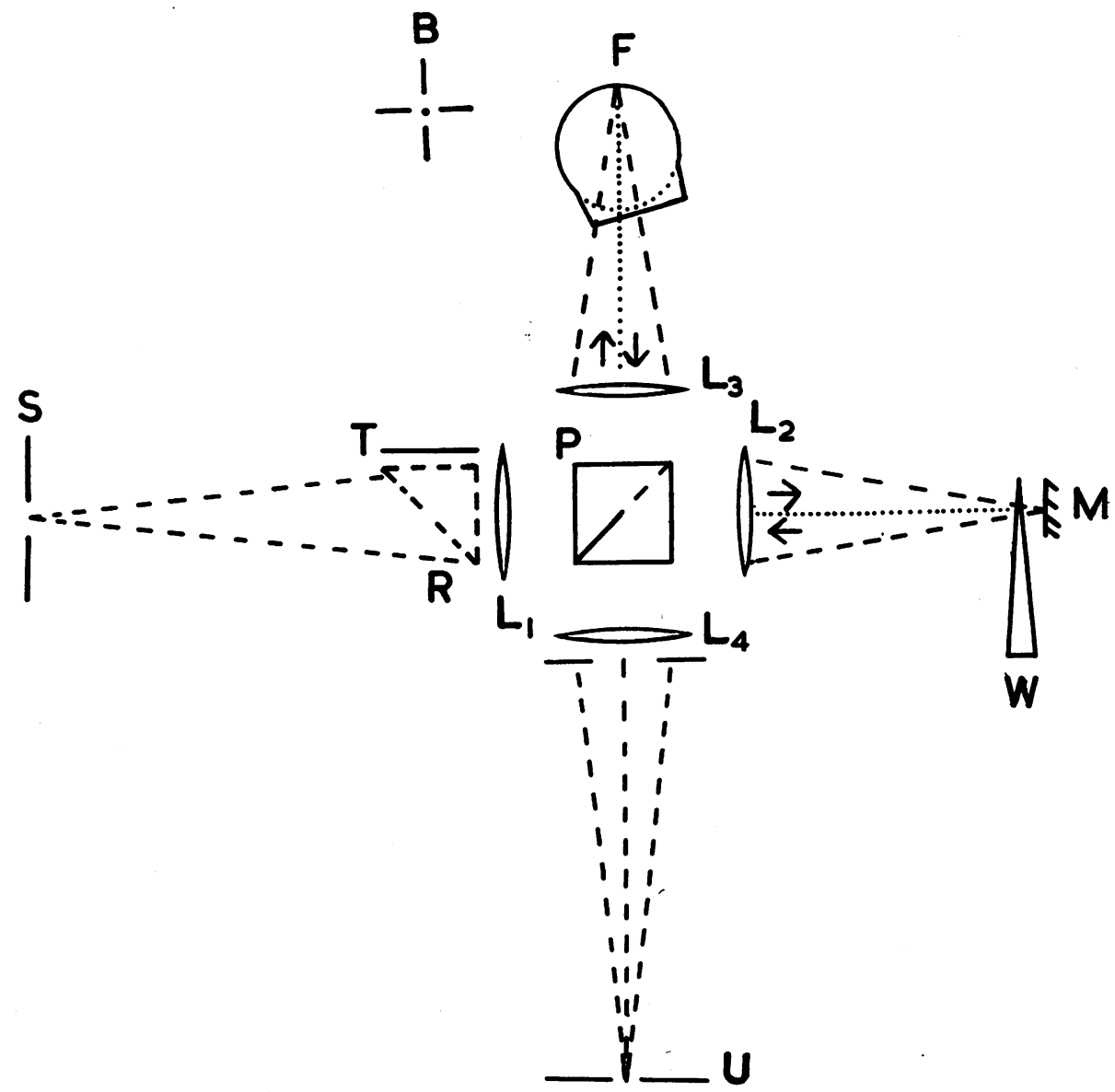

Fig. 1.-Diagram of apparatus (not to scale): Method A. 
of the prism $P$, and focused by $L_{3}$ onto the animal's fundus $F$. Each beam returned after reflection at $M$ and $F$ respectively along a path adjacent to that of its parent. Consequently, the beam from $\mathrm{M}$ could be reflected by the posterior face of the prism mirror at $P$, and the beam from $F$ could by-pass it. Hence, the two beams were juxtaposed and brought to a common focus $U$ by the lens $L_{4}$. If an observer's pupil coincided with this focal point he saw lens $L_{4}$ in Maxwellian view. Use of the neutral density wedge $\mathrm{W}$ in the path of the matching beam enabled him to equate the luminances of the two beams at any wavelength. The apparatus was calibrated in a standard manner ( $c f$. Weale, 1953c).

$B$ was a 12 v $48 \mathrm{w}$ car lamp. The reflecting prism $R$ made it possible to focus its filament on the animal's fundus and to bleach the retinal photo-sensitive material. The intensity and spectral distribution of the bleaching beam could be controlled by suitable filters placed at $T$ ( $R$ was removed during the act of measurement).

A cat, anaesthetized with pentobarbitone sodium, decerebrated, and curarized, was positioned in front of a reflectometer as previously described (Weale, 1953c). The eye was exposed to white bleaching light $\left(\mathbf{S}_{\mathrm{A}}\right)$. In the present experiments, the luminance in the plane of the pupil was approximately $57,000 \mathrm{~mL}$. Since the ocular refraction had been neutralized with a contact lens (Weale, 1953b), and the transmission of the cat's crystalline lens is high, this is the retinal illumination to a first approximation. When the light was switched off after 3 minutes, the reflexion factor $R_{\lambda}$ was measured every minute with light of one particular wavelength. In general, the rate at which $R_{\lambda}$ decreased was consistent with the view that visual purple was regenerating. In some animals, however, the rate of regeneration was uncommonly fast (Weale, 1953a, b). This necessitated more frequent observations. In this manner, it was possible to obtain a regeneration curve (change in density $\Delta D=-\Delta \log R_{\lambda}$ ) for several wavelengths which reached a maximum after about 1 minute. A plateau having been reached, the eye was exposed to the bleaching light for the same period as before (when the eye reacted "rapidly", the bleaching period was reduced to 1 minute) and $R_{\lambda}$ was redetermined after the light had been switched off. The data were accepted only if the value agreed with the initial value previously obtained. This procedure was repeated for various test wavelengths. Hence a given cat could be examined with test-lights of several wavelengths in turn, control measurements at a given wavelength included.

The experiments were extended to frogs (Rana temporaria), dark-adapted during the night, and pithed and decerebrated in deep red light. Rather than embark on the prolonged measurements of re-accumulation as had been done in the cat (Weale, 1953b), the intention was to measure bleaching spectra such as those obtained for guinea-pigs and grey squirrels (Weale, 1955a, b). When the animal had been rendered immobile, it was immersed in a can filled with water, so that its left eye came to be placed near a vertical plane glass window (Weale, 1956b). This arrangement facilitated both the neutralization of the corneal refraction and temperature control. When $R_{\lambda}$ had been measured for the dark-adapted eye, the latter was exposed to white light $(1,450 \mathrm{~mL})$ for $30 \mathrm{sec}$., and $R_{\lambda}$ was redetermined. Surprisingly, a rapid effect-shown by a fast reduction of $R_{\lambda}$-was observed. Again, it was measured for several wavelengths and controls were done at a given wavelength. $R_{\lambda}$ is lower in the frog than in the cat; hence these measurements were more difficult to obtain.

The migration of the pigment granules of the retinal epithelium did not interfere with these measurements which were complete long before it had become appreciable. One of the two eyes of a dark-adapted frog was exposed to the bleaching light under the same conditions as those which prevailed during the previous experiments. The eyes were 
then excised, immersed in Kolmer's fixative for cold-blooded material, and embedded in wax. $8 \mu$ sections were then cut, and stained with Azan stain. There was no visible difference between the sections from the exposed and the unexposed eyes respectively.

\section{Method B}

The above experiments made it desirable to record the whole spectrum rapidly. It takes a long time to obtain measurements at a sufficient number of wavelengths to yield a picture of the photochemical processes involved. The present spectrophotographic method was designed to minimize this difficulty. Disregarding the time of dark-adaptation, even a detailed experiment can be completed in 4 to 5 minutes. (A price for this saving, however, is paid in the subsequent densitometric analysis). Although another technique, now being developed, is superseding the spectro-photographic method, the results obtained with the latter are of sufficient interest to merit consideration.

(a) Apparatus.-Light from a Xenon arc A (type XBO162) was collected by the lens $L_{1}$, and passed through a shutter $S$ (Fig. 2) and the cell C. The right-angled prism $P_{1}$ (the hypotenuse of which was silvered and protected with shellac) deflected the beam into the animal's eye. The dioptric apparatus of the latter was neutralized by means of a contact lens as before. The achromatic lens $\mathrm{L}_{2}$ imaged the fundus on the lower part of the entrance slit $\mathrm{E}$ (divided horizontally by means of a metal strip) of a Hilger spectrograph. A spectrum was thus formed on the ground-glass screen or photographic
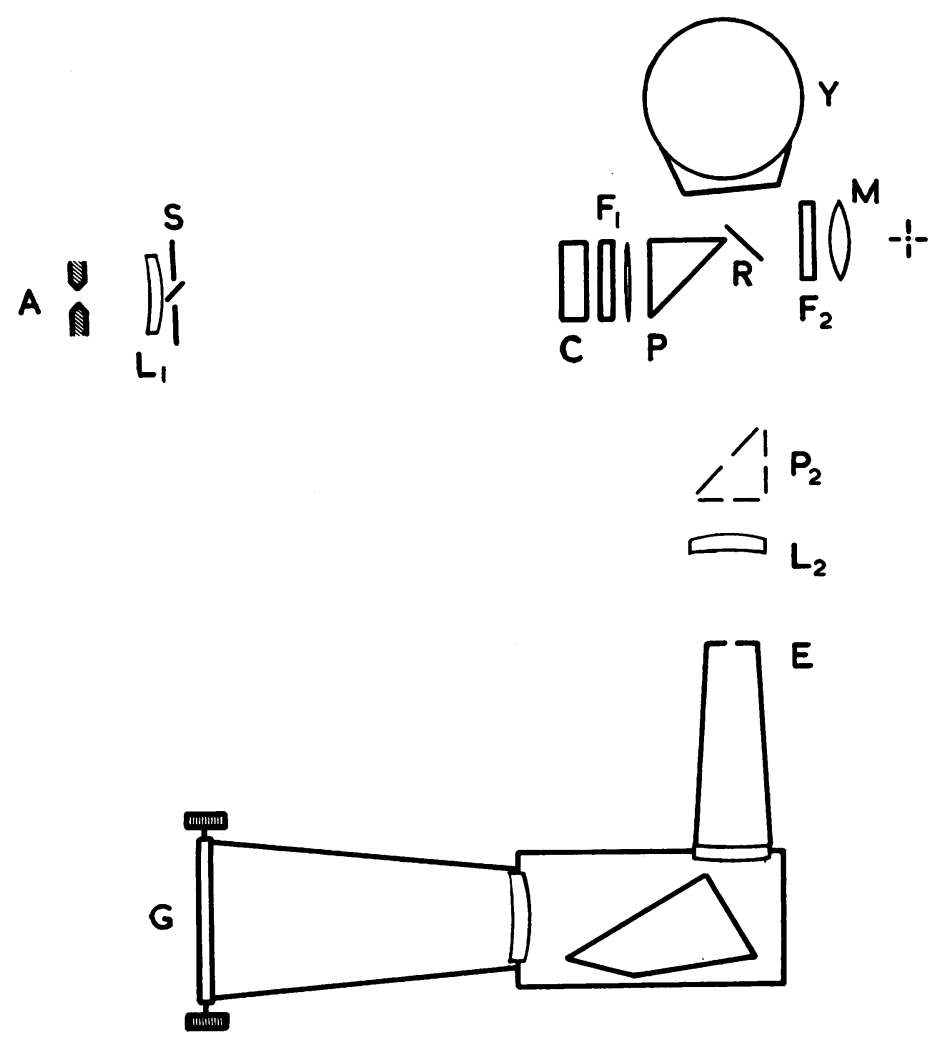

Fig. 2.-Diagram of apparatus (not to scale): Method B. 
plate at G. The original telescope lens of the spectrograph was replaced with one having a shorter focal length in order to increase the luminance of the image.

A plane mirror, placed above the animal's eye at $Y$, reflected a portion of the incident beam onto the upper half of the slit $\mathrm{E}$. This beam provided a monitoring spectrum, variations in the intensity of which were due solely to fluctuations in the Xenon arc. Density changes on the photographic plate, due to this cause, could therefore be compensated.

The angle subtended by the lens $L_{1}$ at the animal's pupil was $3^{\circ}$. By virtue of the narrowness of the entrance slit only a much smaller but undetermined retinal area was examined. This may be thought to be an inefficient arrangement, unless one considers that the governing factor is the adequate exposure of the photographic plate. Exposing a retinal area which is not photographed cannot affect the record. If an optical arrangement were used to concentrate the surplus light into the area which is photographed, the density of the filter $F_{1}$ would have had to be increased to reduce the exposure.

The effective slit width was $3.3 \mathrm{~m} \mu$ at $480 \mathrm{~m} \mu, 5.9 \mathrm{~m} \mu$ at $546 \mathrm{~m} \mu$, and $9.4 \mathrm{~m} \mu$ at $644 \mathrm{~m} \mu$ in the experiments on cats, and was increased by 50 per cent. in those on rabbits.

For bleaching purposes, use was made of the Xenon arc or of a subsidiary source, a microscope lamp $M$. A filter $F_{2}$ controlled the spectral distribution of the bleaching light which reached the eye by the movable mirror $R$. The lamp filament was focused on the fundus.

(b) Animals.-Cats and albino rabbits were examined. The cats were anaesthetized with pentobarbitone sodium, decerebrated, and curarized. The rabbits were anaesthetized with urethane, and curarized. Artificial respiration was applied in all cases. Only one animal (a cat) died during an experiment.

(c) Procedure.-The animal's head was firmly secured in a suitable head-clamp and the left eye positioned at Y (Fig. 2). Rabbit fundi are substantially alike; hence, the appropriate photographic exposure had to be determined only once. The variability of the cat tapetum, however, made it necessary in each case to expose and develop a pilot plate in order to arrive at the optimum conditions. This done, the animal was darkadapted for $1 \frac{1}{2}$ hrs. A fundus spectro-photograph was taken and the photographed part of the fundus was exposed to the bleaching light for a timed period. Another spectrophotograph was taken within 2 sec. after the bleaching light was switched off. This course was repeated five or six times, and the final bleaching done. The densities on the plate were then calibrated: neutral density filters of nominal values $0 \cdot 1, \cdot 0 \cdot 21$, and 0.32 were placed in turn at $F_{1}$, and fundus spectro-photographs were taken on the same plate. Finally, line-spectra of a Cd-Hg lamp $\mathrm{H}$ were photographed at the top and bottom of each plate, the reflecting prism $\mathrm{P}_{2}$ having been inserted for the purpose. The plate was developed immediately afterwards. The animal was dark-adapted once again, and, in favourable circumstances, three periods of dark-adaptation and sets of exposures could be obtained from one animal.

(d) Photographic Technique.-The fastest obtainable plates, Kodak P 2000, were used. Their high sensitivity to light of relatively long wavelengths had to be balanced with a red-absorbing filter in order to avoid over-exposure. A 1 per cent. solution of Azan, diluted to $1: 4$ or $1: 8$ and contained in the cell $C$, was used for this purpose. Two pieces of heat-absorbing glass were stuck to the cell. The overall exposure was controlled by a neutral density filter at $F_{1}$. The time of exposure was kept at $1 / 5 \mathrm{sec}$. to minimize the effect of the flicker of the A.C. source. Exposures of 1/50 sec. were feasible but impracticable. The source could not be run off D.C., but the longer exposure time ensured adequate integration.

(e) Densitometry.-All the spectro-photographs were examined with a Hilger microdensitometer. The plate was aligned by means of the two line-spectra at the top and 
bottom respectively. A zero position was then selected in the unexposed region beyond the long wavelength limit for each fundus spectro-photograph and the relative transmission factor of the spectra measured. The vernier scale of the instrument, attached to the screw which moved the plate across the measuring beam, was calibrated in terms of the known wavelengths of the line spectra, intermediate positions being found by interpolation. It was thus possible to measure and to compare the transmission factors of the spectro-photographs on any one plate for any chosen wavelength. The stability of the instrument and the position of the plate were frequently checked during each set of measurements.

The densities of the monitoring spectra were measured at one wavelength only, any variation in this density being the same in different parts of the spectrum. This would be expected except in grossly under- or over-exposed parts of the spectrum. Corrections due to such variations were applied to the fundus spectro-photographs.

Before the difference spectra could be evaluated, it was necessary to plot a calibration curve for each wavelength and plate. This was done by subtracting the densities obtained with the $0 \cdot 1,0 \cdot 21$, and 0.32 filters respectively from the density values of the spectro-photograph corresponding to the bleached fundus. The filters had been calibrated between wavelengths 390 and $650 \mathrm{~m} \mu$ with a Hilger SP 600 spectro-photometer; hence the calibration curves related the plate densities to true densities. The curves were in general rectilinear, the slope agreeing with that obtained for the relevant monitoring spectra. This provided a check for the stability of the fundus while the calibration photographs were taken.

The plate density differences-always referring to the "fully bleached" spectrum-were then calculated, the true density differences were read off the curves, and difference spectra were plotted in the usual manner. Examples of "dark-adapted" and "fullybleached" spectrophotographs (rabbit) are shown in Fig. 3.

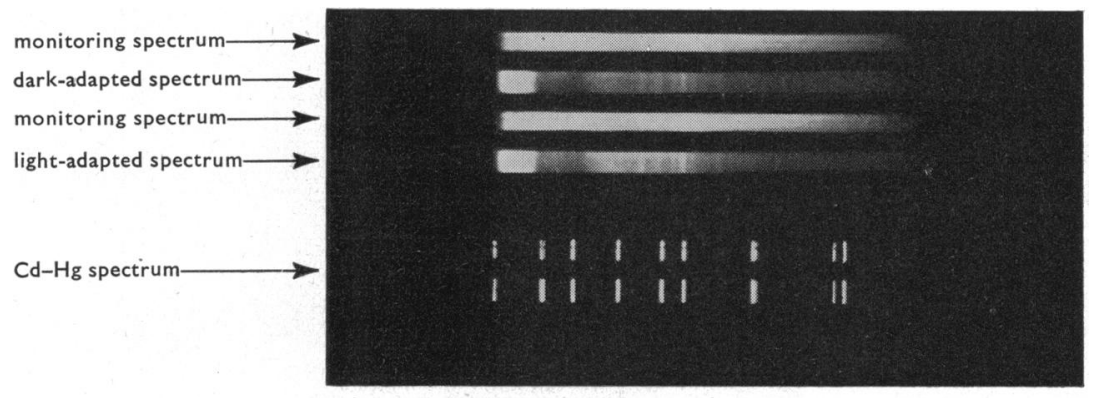

FIG. 3.-Spectrophotograph obtained for a rabbit fundus. The long spectra monitor the intensity of the source of light.

Top: First photograph after $1 \frac{1}{2}$ hrs dark-adaptation.

Bottom: Final photograph after bleaching with white light.

The line spectra were obtained from a Cd-Hg lamp.

(f) Type of Experiments Performed.-The factors which varied in these experiments were the duration, intensity, and spectral distribution of the bleaching light. The effect of a single flash used in taking the photographs was measured. White light of varying intensities and durations was also used. Chromatic partial bleaches were done by placing different Ilford filters in turn at $\mathbf{F}_{2}$. In all cases, however, the experiment was completed by bleaching with white light of full intensity. The study of dark reactions was vitiated by complications due to regeneration. Photometric measurements of the luminances employed are shown in the Table (opposite). 
TABLE

LUMINANCE ( $m L$ ) IN THE PLANE OF THE ANIMAL'S PUPIL

\begin{tabular}{|c|c|c|c|}
\hline Fig. 6 & \multicolumn{2}{|l|}{ Photographic Exposure } & 710 \\
\hline Fig. 7 (a) Rabbits .. & \multicolumn{2}{|c|}{$\begin{array}{l}\text { Photographic Exposure } \\
\text { White Bleaching Light (microscope lamp M) }\end{array}$} & $\begin{array}{l}3,300 \\
7,600\end{array}$ \\
\hline \multirow[t]{2}{*}{ Fig. 7 (b) Cats } & \multirow[t]{2}{*}{ Photographic Exposure } & *Plate Nos. 69, 77, 78, 111 & 330 \\
\hline & & †Plate Nos. 96, 97 & 225 \\
\hline
\end{tabular}

\begin{tabular}{|c|c|c|c|}
\hline Plate No. & Bleaching History & Final Bleaching Light & $\begin{array}{c}\Delta \mathrm{D}_{\max } \\
\text { (cf. Fig. 8) }\end{array}$ \\
\hline$\dagger \quad 96$ & $\begin{array}{l}1,380 \mathrm{~mL} \text { min. White (M) } \\
+6,480 \mathrm{~mL} \text { min. (arc) }\end{array}$ & & \\
\hline$\dagger \quad 97$ & $\begin{array}{l}910 \mathrm{~mL} \text { min. Orange (Ilford 607) } \\
+17,500 \mathrm{~mL} \text { min. White (M) } \\
+6,480 \mathrm{~mL} \text { min. (arc) }\end{array}$ & & \\
\hline * 69 & $\begin{array}{l}21 \mathrm{~mL} \text { min. Violet (Ilford 601) } \\
+8,750 \mathrm{~mL} \text { min. White }(\mathrm{M})\end{array}$ & $8,750 \mathrm{~mL}$ min. $(\mathrm{M})$ & $0 \cdot 120$ \\
\hline * $\quad 77$ & $\begin{array}{l}520 \mathrm{~mL} \min . \text { Red (Ilford 608) } \\
+8,750 \mathrm{~mL} \text { min. White (M) }\end{array}$ & $8,750 \mathrm{~mL}$ min. $(\mathrm{M})$ & $0 \cdot 118$ \\
\hline * 78 & $9,480 \mathrm{~mL}$ min. White (M) & $8,750 \mathrm{~mL}$ min. (M) & 0.092 \\
\hline * 111 & $200 \mathrm{~mL}$ min. Green (Ilford 604) & 6,480 mL min. (arc) & $0 \cdot 120$ \\
\hline
\end{tabular}

* Fig. 7

The following experiment was performed in order to discover whether the exposure of the dark-adapted eye to intense light produces any vascular changes in the cat fundus.

The apparatus shown in Fig. 2 was modified by substituting a reflecting for the dispersing prism, and a microscope cover-slip for the slit. The cover-slip was so adjusted as to allow one of its horizontal edges to coincide with the central part of the fundus image. Fundus photographs were taken on Kodak P 600 plates. The animals having been dark-adapted for at least 1 hour, a photograph was taken with light from the microscope lamp M. After 2 minutes' exposure of the eye to light from $\mathbf{M}$, another photograph was taken on the same plate, and so on until the eye had been illuminated continuously with the undimmed light for $\mathbf{1 0}$ minutes. As the right femoral artery had been cannulated, a check could be kept on the animal's systemic blood pressure. When the plate was developed, density measurements were made along the line provided by the image of the edge of the cover-slip. Veins and arteries had been marked on a drawing; it was thus possible to associate density changes on the plate with each vessel. Micrometric control enabled one to compare photographs of the widths of any given vessel.

Although a change as small as 3 per cent. could have been detected, no systematic changes were observed, presumably because they did not occur. It would appear, therefore, that, under the conditions of the present experiments, vascular changes are not likely to mask other effects due to exposure of the eye to light. 


\section{Results}

All the density changes represent the measured changes, i.e. the values have not been halved as should be done if, after reflection at the fundus or tapetum, every beam retraced its optical path through the retina. The data for rabbits have been corrected for the reduction in the measured density change caused by the light scattered by the pre-retinal media. The results obtained for the eyes of albino guinea-pigs (Weale, 1955a) were used for this purpose. The high reflection factor of the cat tapetum (Weale, 1953c) allows one to neglect a similar correction to the data for cats. The correction for the frog eye could not be determined on account of its small size. Data obtained for pigmented guinea-pigs, however, suggest that, in the spectral region here covered, such a correction can be neglected.

(a) The Rapid Effect.-A typical curve obtained from a cat has been previously published (Weale, 1953b) and is here reproduced for the sake of completeness. An analogous curve obtained from a frog eye is also shown (Fig. 4).

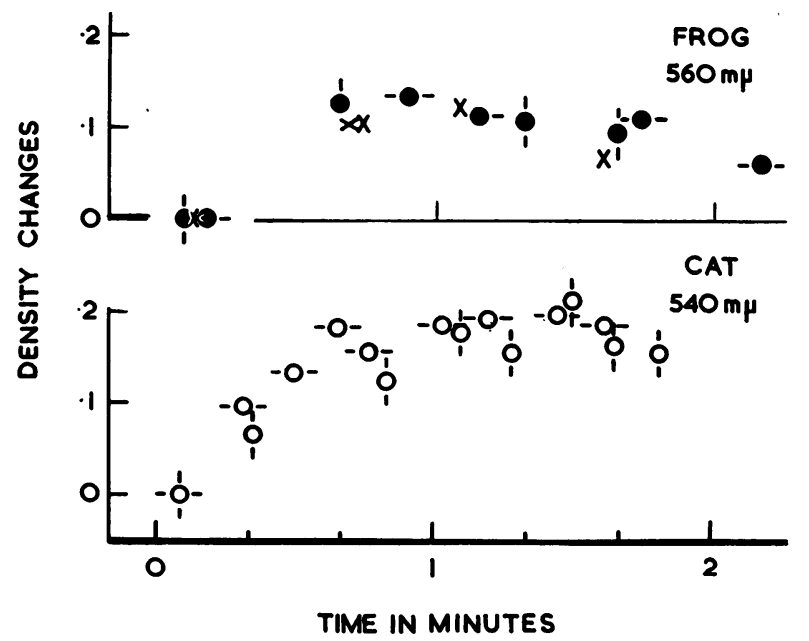

FIG. 4.-Examples of "rapid effect".

Top: Frog. Test-light: $560 \mathrm{~m} \mu$. First run: $\theta ; 3$ minutes later: $\Phi$; 48 minutes later: $X$; 2 minutes later: $X$.

Bottom: Cat. Test-light: $540 \mathrm{~m} \mu$. First run: $\Phi$; 10 minutes later: $\vartheta$.

The reduction in the density increase after about one minute's observation will be noted. Beyond the maximum of the curve there was a tendency for the fundus reflectivity to increase. Indeed, when cat eyes showing the rapid effect were exposed to the test-light for longer than necessary, $R_{\lambda}$ increased visibly. This suggests that radiation as weak as that of the monochromatic test-light ( $c f$. Weale, 1955a) is sufficiently intense to counteract the effect of regeneration. The spectral distribution of the effect (Fig. 5, opposite) has been obtained by measuring 


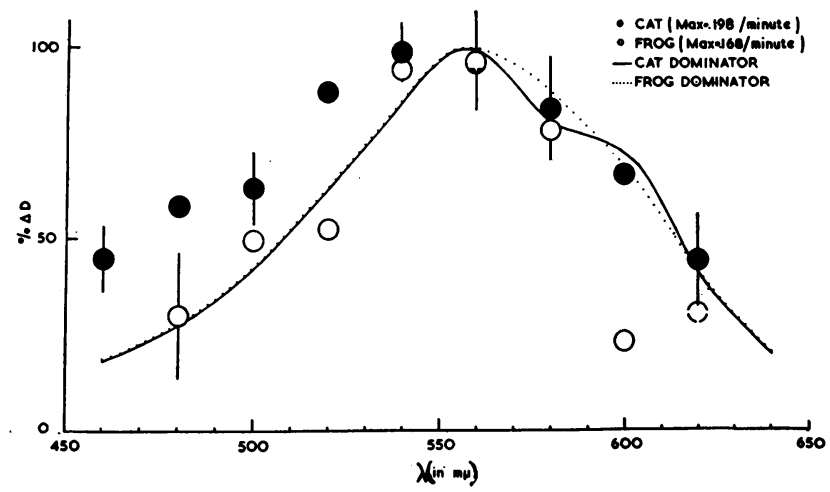

FIG. 5.-Initial rates of "rapid effect".

Black circles average of experiments on six cats (Max. $=0 \cdot 198 /$ minute).

White circles average of experiments on four frogs (Max. $=0 \cdot 168 /$ minute).

Broken symbols indicate that only one datum was available.

Full and dotted lines represent photopic dominators found in cat and frog retinae respectively (Granit, 1947).

the initial rate of the density at each wavelength. The spectral distributions obtained for the cat and frog are remarkably alike. Sample standard errors of the mean are shown.

(b) Effect of a Single Flash on a Dark-Adapted Eye.-An example is shown in Fig. 6 for a cat. Analogous data for the rabbit, obtained with higher intensity flashes, showed a greater initial change with a spectral distribution indicating some loss of visual purple.

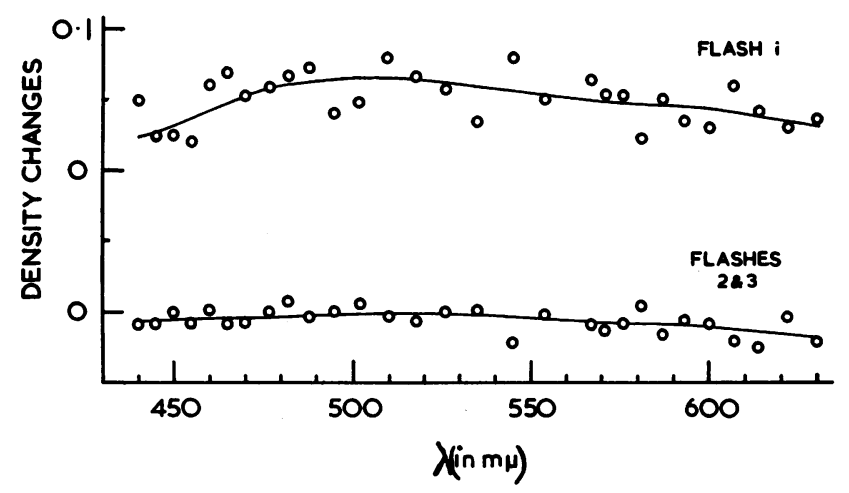

FIG. 6.-Effect of photographic flash. For details see text.

(c) Total Bleaches.-The averages of three total bleaches done on two rabbits, and of six such bleaches done on four cats, are shown in Fig. $7 a$ and $b$ (overleaf). As the wavelengths at which measurements were made were not generally the same, the data were grouped into wavelength ranges of width $5 \mathrm{~m} \mu$ for $\lambda<520 \mathrm{~m} \mu$, 


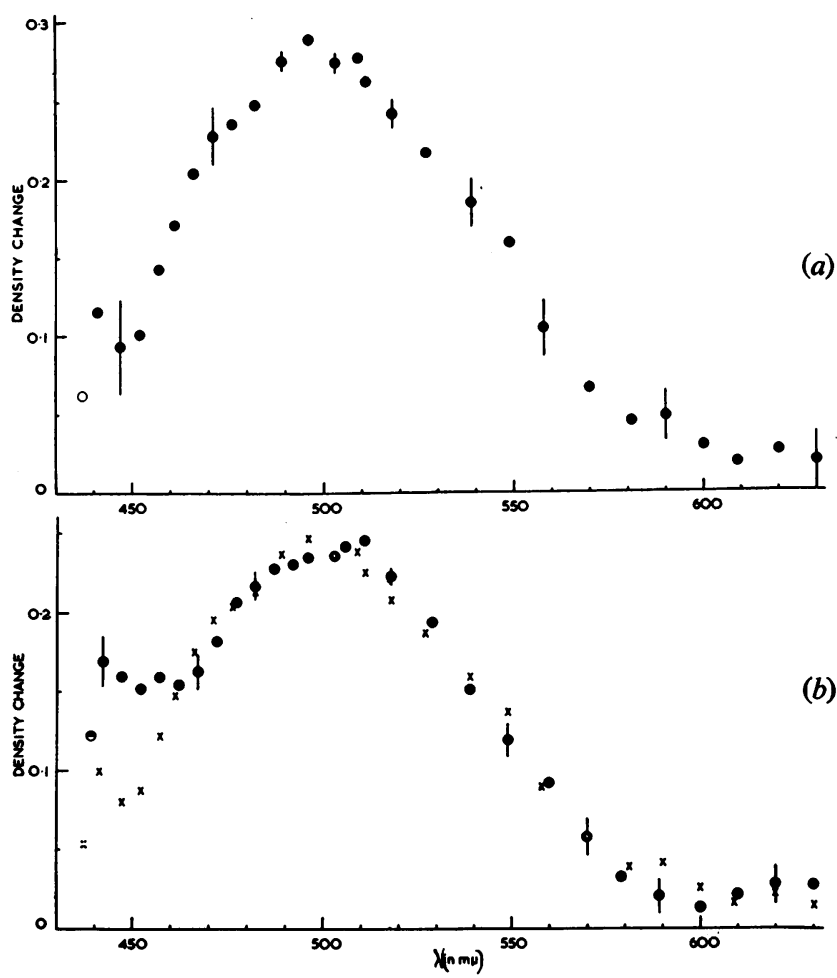

FIG. 7 (a).-Mean of three " total bleach" difference spectra (two rabbits).

FIG. 7 (b).-Mean of six " total bleach" difference spectra (four cats).

The maxima of the individual difference spectra were set equal to 100 and the other points calculated as explained in the text.

Broken symbols indicate that only one reading was available.

Rabbit data from (a) reduced in scale (X) to facilitate comparison between the two sets of data.

and of width $10 \mathrm{~m} \mu$ for wavelengths longer than this. The data on the rabbits, suitably reduced along the ordinate, are replotted as crosses (X) in the lower part of the diagram to facilitate comparison between the results for the two species. Except for the blue part of the spectrum they agree within the experimental error. Examples of the standard error of the mean are shown.

(d) Final Bleaches.-A remarkable feature of this study was the finding of a change during (though not necessarily due to) the final white bleach. The difference spectrum was substantially independent in shape and magnitude from the previous bleaching history of the eye. The mean of four such difference spectra (obtained from cats) is shown in Fig. 8 (opposite). The shallow curve has a maximum significantly displaced from $500 \mathrm{~m} \mu$ (where it would be expected if visual purple were disappearing) towards shorter wavelengths $(\approx 485 \mathrm{~m} \mu)$. 


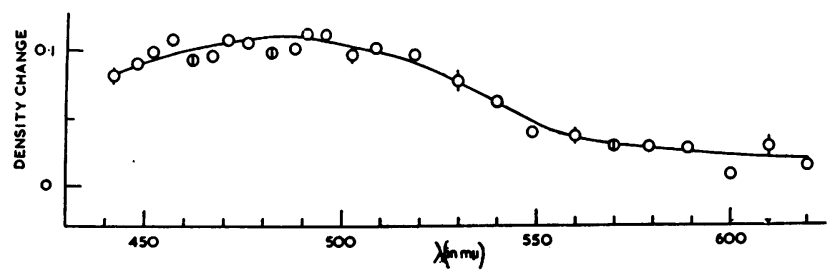

FIG. 8.-Average of four final "white bleach" difference spectra.

\section{Discussion}

(a) Absolute Density Values.-Hagins and Rushton (1953) showed that, for a rabbit eye, the ratio between the density change, as measured by light reflected from the fundus, and that found by measuring the light transmitted through the sclera is 2 . Lewis (1956), working on rat eyes, found a ratio of 1.86. The error in using a value of 2 is, therefore, small (Weale, 1953b, 1955a), but may be serious when cone pigments are examined (Weale, 1955b). Nonetheless, provided the position is clearly stated, it is better to quote absolute values, subject to whatever correction, than to allow information to be lost by quoting relative values only. Several factors lead to an underestimation of the true density changes:

(i) Scattered light; light received which has not passed through photo-sensitive material either because it was reflected before reaching the rods and cones, or because it slipped through between them on both journeys through the retina;

(ii) Light which has missed rods and cones only on one journey;

(iii) Regeneration of the bleached material during measurements;

(iv) Bleaching of the pigment by the test beam.

Thus the absolute density values shown in Figs 4 to 7 , which refer to the measured, as distinct from a calculated, density change, must be considered to be minimal (this stricture applies generally to photometric measurements of density changes in light-sensitive pigments).

(b) The Rapid Effect.-Granit (1955) has suggested that the fast effect in the cat may underlie the photopic dominator, which he revealed by means of his micro-electrode technique. In this connexion it is of interest to note that light-adapted frog eyes yield similar dominator curves. Again, in none of over one hundred bleaching experiments done on guinea-pig eyes, has there been any indication of any rapid effect, nor has a photopic dominator been obtained from guinea-pig retinae. These three species thus support Granit's suggestion. Evidence for the rapid effect was also obtained photographically. But, by its very nature, Method B does not lend itself to the detailed study of this phenomenon, and hence no attempt was made to obtain a thorough quantitative evaluation of these results.

The observations on the rapid effect in the cat have been confirmed by Campbell and Rushton (1955). But, because it was impossible to record an action spectrum 
corresponding to the difference spectrum obtained from bleaching experiments, these authors tentatively suggested that the changes were due to some unspecified tapetal reflex. However, failure to record a given action spectrum hardly justifies rejecting the notion that one is dealing with photochemical changes. The rapid increase in $R_{\lambda}$, ascribed to the regeneration of a photo-sensitive substance (Weale, 1953a), occurs after the eye has been exposed to light and the light has been turned off. If light causes the effect, there must be an action spectrum, whether the effect takes place in the retina, the tapetum, or even the cornea. It may be that the rapidity of the effect makes a correlation between action and difference spectra difficult, unless many animals have been examined. Furthermore, when examining the human fovea, Rushton (1955) observed similar rapid effects, the action spectra of which may well correspond to the sensory counterpart of the photopic dominator (Wright, 1946). A photochemical basis may thus be ascribed to the three effects found in the cat, frog, and human fundi respectively.

(c) Accuracy of the Photographic Data.-Under optimum conditions, two isochromatic radiations can be compared photographically with an accuracy of about 1 per cent. (Forsythe, 1937).

Such a degree of accuracy demands the juxtaposition of the two exposed areas on the emulsion. Again, the uniform development of a plate, such as P 2000, of even moderate size $\left(\frac{1}{4}\right)$ is well-nigh impossible. Thus the desire to obtain a maximum number of fundus spectro-photographs on a single plate and considerations of accuracy impose a compromise which, of necessity, leads to reduced accuracy. This is why the calibration spectra were interspersed with the experimental ones, non-uniformity in the developed emulsion having then a smaller adverse effect on densitometry than if the calibration spectra were concentrated in one place. Precision (important in connexion with the monitoring spectra, the density of which determines the zero line of the difference spectra) was considerably increased by examining four different points at a given wavelength range, i.e. along a line at right angles to the length of the monitoring spectra.

These considerations explain why the scatter of the individual points is of the order of $0.01-0.02$ density units for double transit of the light through the retina (approximately $0.005-0.01$ for single transit).

Personal judgment enters these measurements only to the extent of reading a galvanometer scale. Hence the data are free from the bias which may affect results obtained by zero methods.

(d) Effect of Recording Flashes.-It is noteworthy that, although the first flash causes a small but appreciable change in density, the second and subsequent flashes are without measurable effect. This appears to dispose of the possibility that a small proportion of visual pigment is bleached by these flashes, for if it were one would expect the change to be nearly as great for the second as for the first flash. Although the present observations cannot throw any light on the character of the changes caused by the recording flashes, it seems clear that they are not of the same nature as those caused by the "bleaching" lights. Since the measurements shown in Fig. 6 were recorded within a few seconds of each other, it may be that these density 
changes represent the difference between the light-absorbing properties of inactivated and activated visual purple respectively (cf. Wulff and others, 1956). A possible alternative is that the light used for the photographic exposure, while too weak to bleach a measurable amount of visual purple at any rate in the cat, does remove the "rapid" substance. Its subsequent regeneration may be impeded by further photographic and bleaching exposures. Whatever the ultimate explanation of these findings, the fact that the second and third flashes do not record any change lends support to the view that the subsequent changes are due to effects caused by the bleaching lights rather than to others due to the reçording flashes.

(e) Chromatic and White Bleaches.-Results obtained with partial white bleaches, other than the final, are consistent with the view that the principal pigment to disappear is visual purple. These bleaches were done with a weaker light than was usual in the original experiments on the cat (Weale, 1953b); this accounts for the slight discrepancy between the old and the new data.

A comparison between the results for total bleaches carried out on cat and rabbit eyes respectively suggests that the two types of eye differ mainly in the relative amounts of a blue-sensitive substance (cf. Dodt and Elenius, 1956) possibly present in the cat retina. Evidence for such a substance had previously been obtained for the cat (cf. Granit, 1955) by the same method as was used for guinea-pigs (Weale, 1955a). While partial violet bleaching yielded confirmatory evidence in the present set of experiments, the interpretation of the data is rendered more difficult by the occurrence of the change shown in Fig. 8.

$(f)$ Final White Bleaches.-There is a marked similarity between the difference spectra recording changes which occurred while the final white bleaches were taking place. They are independent of the spectral composition of the light with which the earlier bleaches were effected, and this suggests that they form part of the bleaching process of visual purple itself. Both in its spectral shape and in the position of its maximum, the spectrum resembles that of meta-rhodopsin (Wald, Durell, and St. George, 1950). This substance has previously been characterized in vitro. It is, therefore, gratifying to find that some of the effects, dismissed perhaps as mere in vitro phenomena, find their analogues in the living eye.

\section{Summary}

A photometric method, previously described, was used for the measurement of changes in fundus reflectivity, $\boldsymbol{R}_{\lambda}$, in living eyes. After exposure to white light, cat fundi sometimes showed a rapid decrease in $R_{\lambda}$, which suggested the accumulation of a light-sensitive retinal substance. When frogs were examined in a similar way, a change in $R_{\lambda}$ was observed, which, 
both in its spectral distribution and in its rate, was similar to that found in cats. It is suggested, following Granit (1955), that these effects underlie the photopic dominators observed by electro-physiological means. The desirability of more rapid overall spectral recording followed from these findings, and a spectrophotographic method is described which was applied to living cat and rabbit eyes. Bleaching lights of various intensity, duration, and spectral composition were employed in the analysis of the cat's photosensitive material. The most striking finding was the occurrence of a secondary change which is spectrally similar to the meta-rhodopsin of Wald, Durell, and St. George (1950).

I should like to thank Prof. B. K. Johnson, Imperial College, University of London, for permission to use a microdensitometer for the examination of some pilot plates, also Dr. K. Tansley of the Institute of Ophthalmology, and Dr. W. A. H. Rushton, Cambridge, for valuable discussions, and Miss G. Villermet for her enthusiastic help during the experiments.

\section{REFERENCES}

CAmpbell, F. W., and Rushton, W. A. H. (1955). J. Physiol. (Lond.), 130, 131.

DODT, E., and ELENIUS, V. (1956). Pfügers Arch., 262, 301.

FORSYTHE, W. E., ed. (1937). "Measurement of Radiant Energy". McGraw-Hill, New York and London.

GranIT, R. (1947). "Sensory Mechanism of the Retina". Oxford Univ. Press, London. (1955). "Receptors and Sensory Perception". Yale Univ. Press, New Haven.

HAGINS, W. A. (1956). Nature (Lond.), 177, 989. and Rushron, W. A. H. (1953). J. Physiol. (Lond.), 120, 61P.

LEWIS, D. M. (1956). Ibid., 133, 55P.

RUSHTON, W. A. H. (1955). Farbe, 4, 219.

WALD, G., Durell, J., and St. GeORGE, R. C. C. (1950). Science, 111, 179.

WEALE, R.A. (1950-1). "Institute of Ophthalmology. Third Annual Report, 1950-1951", p. 18.

- (1953a). J. Physiol. (Lond.), 122, 11P.

- (1953b). Ibid., 122, 322.

- (1953c). Ibid., 119, 30.

(1955a). Ibid., 127, 572.

(1955b). Ibid., 127, 587 .

(1956a). " "20th Internat. Physiol. Congr., Brussels, 1956", Abstracts of Communications, p. 951 .

(1956b). J. Physiol. (Lond.), 132, 257.

WRIGHT, W. D. (1946). "Researches on Normal and Defective Colour Vision". Kimpton, London.

WulfF, V. J., Abrahamson, E. W., Adams, R. G., and Linschitz, H. (1956). " 20 th Internat. Physiol. Congr., Brussels, 1956," Abstracts of Communications, p. 979. 